

\title{
PLASMAS IN PARTICLE ACCELERATORS: ADIABATIC THEORIES FOR
} BUNCHED BEAMS

\author{
James H-K. Mark \\ Lawrence Livermore Mational Laboratory
}

March 3, 1981

Abstract

Three different fonalisms for discussing Vlasov's equation for bunched beam problems with anharmonic space charge forces are out lined. These correspond to the use of a drift kinet ic equation averaged over random betatron motions; a fluidkinetic adfabatic regine analogous to the theory of Chew, Goldberger, and Low; and an adiabat ic hydrodynamic theory.

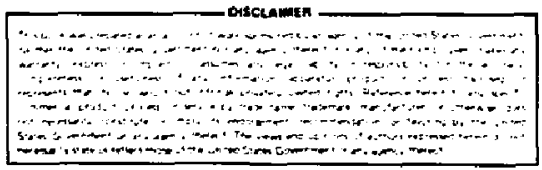


I. Introduction

Induction linear particle accelerators are being designed for application as orivers for heavy-ion inertial fusion energy power plants [1-6]. As part of the process, it is necessary to identify and aroid regimes of longitudinal instability in bunched beams. Discussions of these problems $[1-4,6]$ have gersally been pursued using inf initely thin beans. The question of transverse structure in these instabilities has not been considered. Even though these longitudinal instabilities are usually axisymmetric, introduction of the transverse dimensions results in rather complicated three dimensional orbits for the individual particies. Especially in the presence of anharnonic transverse forces. the transverse motion are rosette orbits in a central force. The solution of Ylasor's equaticns (cf. Ref. [7]) involves the three dimensional effects of the betatron transverse orbit oscillations. A treatment including two spatial dimensions, three velocity cnes and the time parameter might seen necessary. One purpose of this paper is to provice mathematical formalisms which show that the solution of Vlasov's equarion for this problem can be reduced to two spatial dimensions and one in velocity (Sections $I I$ and III), or even to just two spatial dimensions (Section IV), within physical regimes which allow appropriate asymptotic ordering of parameters.

The discussion is based on the "wartm-beam model" recently described by Mark and Snith (Ref. [8]; hereinafter referred to as Paper I). Namely, we take the point of view that the forces on the particle are governed by an axisymetric averaged component $f_{a y}(r, c z, c t)$ perturbed by a 
smaller fluctuating part $F_{f \ell}(r, \theta, z, t, E t, \ldots)$, where $(r, \theta, z)$ are cylindrical polar coordinates with $z$ axis dlong beam axis and $t$ is the time. The quantity $c$ is a small parameter which indicates, for example, that the force $F_{\text {av }}$ varies more rapidly in the radial direction than the axial one, being directly related to the fact that beam bunches are much longer than they are wide. The time variation in $F$ av is also expected to be slower than that of transverse betatron time $(2 \pi / N)$ of the particles, thus the $(c t)$ dependences.

As a first approximation, we already discussed in Paper 1 the problem of the "coasting-bean" equilibria in the warm beam limit with $F_{\text {av }}(r)$ only. The study of the orbits in the presence of strong anharmonic space charge forces as outlined there is now the starting point of our discussion of bunched baans. We allow in principle not just the external bunching forces, but also internal ones (eg. waves) but stipulate that they fit into the reasonable scheme where forces like $F_{a v}(r, c z, \varepsilon t)$ prevail, while those involving $F_{f \ell}(r, \theta, 2, t, \epsilon t, \ldots)$ are at least $0(\varepsilon)$ smaller. Under these expected conditions where the dominant forces yary little over the betation orbit time, we derive a "drift-kinetic" version of Vlasoy's equation (Eq. II.23) in jection II where some effects of betatron orbits are averaged out. For axisymetric problems, this is a "1-1/2 dimension" equation describing exact dynanics in the axial direction, but providing an approximate description of the transverse cirections to determine self-consistent forces. In this equation (11.23), a11 that remains of the transverse motions (for axisymmetric problems) is an averaged radial (hydrodynamic) drift (one reason for the name "driftkinetic equation"). 
A more accurate set of equations can be obtained (cf. Section III) under conditions similar to the so-called "adiabatic fluid-kinetic theory" of Chew, Goldberger and Low [9]. It is important to emphasize that we do not have axial magnetic fields in our problem, so that only careful analyses of particle orbits (such as those of Paper I) exhibits the analogy to the latter theory. The betatron vortex ascillations of Paper I are the analogue of the Lanmor gyrations in an axial magnetic field. Within this adiabatic theory, the exact cont inuity and momentum moments (Eqs. IIi.12-III.15) of Vlasov's equations are used, wile the longitudinal heat $f l c w$ is governed by the above ment ioned drift-kinet ic equation (1I.23).

Sonet imes the adiabatic theory is not trivial to apply in practice, thus it is useful to note (Section IV) that an additional "adiabatic (fully) hydrodynamic theory" obtains if we further assume that part of the longitudinal forces are somewhat. weaker than the ordering allowed in the earlier adiabatic fluid-kinet ic regime. This approximation is equivalent to an additional requirenent on the longitudinal length scale of beam bunches (or disturbances thereof) being much larger than the bean radius. (We note that the bean radius to length $\sim 1 . / 50$, and in any case, the majority of particles barely make it across the beam length). Within this fully hydrodynamic regine, the moment equations for continuity and monent um are now closed by equations relating the stresses to density and velocity so that the description is by equations (III.12)-(1II.15) with closure relations (IV.1) - (IV.3). An alternate just if ication for similar approximations following the "double-adiabatic" arguments of Chew et a1. [10] might be feasible. In this case we presume fdetailed justification has yet to be made) that the 
fluctuating force component $F_{f e}$ provides a field which "collides" sufficiently strongly with the longitudinal particle motion so as to sufficiently inhibit longitudinal heat flow in the bean bunch. Thus heat flow terms in the equations for stresses can be omitted.

Since we have retained full three dimensionality in the derivation of all our equations, the theory can be used to study three-timensional instabilities. For example, the hydrodynamic equations (111.12)-(111.15) with closure relations (IV.1) - (IV.3) could be a justification for the fluid mocel used by Mark, Krafft and Wang [11].

\section{The Drift-Kinetic Equation}

For the present time dependent bunched beam problem we assume, for simplicity, that the averaged accelerating, focusing, bunching and space charge forces are represented by

$$
\frac{1}{m} F_{a_{v}}=-\hat{z} \frac{\partial V_{a}(Z)}{\partial Z}-\nabla V(r, \theta, z, t),
$$

where we now distinguish the $z$ coordinate to be relative to the beam bunch which has an average position $2(t)$ accelerating according to $Z=-a v_{a} / 2 Z$. ( $m$ is the mass of the ions). Rather than explicitly displaying the small parameter $c$ as in Section $I$, we now simply understand that relative to this average beam frame

$$
\varepsilon=O\left(\frac{\partial}{\partial t}\right)=O\left(\frac{\partial}{\partial \theta}\right)=O\left(\frac{\partial}{\partial z}\right) \text {. }
$$

For simplicity of. presentation in Secciors III-IV, we on it for the time being any discussions of magnet:ic fields and electromagnetic problems. 
Using the corresponding momenta per unit mass $\left(p_{r}, p_{\theta}, p_{2}\right)$, we can write the equations of motion (relative to the beam bunch) as

$$
\begin{gathered}
\frac{d r}{d t} \equiv \dot{r}=p_{r}, \quad \dot{p_{r}}=\frac{1}{r^{3}} p_{\theta}^{2}-\frac{\partial V}{\partial r}, \\
\dot{\theta}=\frac{1}{r^{2}} b_{\theta}, \quad \dot{p_{\theta}}=-\frac{\partial V}{\partial \theta}, \\
\ddot{z}=-\frac{\partial V}{\partial z},
\end{gathered}
$$

where the dots atove the symbols indicate tine derivatives. Vlasov's equation in these coordinates is well known.

In addition to the adiabatic assumptions (11.1)-(11.2), we inc rrpurate also the ordering scheme corresponding to the virm-beam equilibria of Paper I. Naneiy, we assune that the presence of nonlinear space charge forces precludes the separate discussion of arbits in two orthogonal transverse directions (thus, our use of $r, 0,2$ coordinates). And, in particular, we specify that typical orbits have

$$
\left|\frac{r p_{r}}{p_{\theta}}\right| \sim \varepsilon \sim\left|\frac{\delta r}{r}\right|<1,
$$

which describe rosette orbits in the anhamonic averaged focusing forces. These orbits have radial excursions or smaller than their typical radius ef. Figs. $1-x$ of Paper $I$ ). 
Following Paper I, we may presume that there are two superposed counter-rotating beam components with areraged circular frequenc ies $\pm|u(r, \theta, z, t)$,$| . For each camponent, we transform vlasov's equation$ into "drift-kinet ic" form by isolating quantities that are our present ana logues of magnetic moment, etc.. This transformation is achieved first through the momentum variables. He introduce the amplitudes $\alpha, \beta$ and phase $*$ of the betatron-vortex oscillations (cf Paper I, Eqs. 14-21) through

$$
\begin{aligned}
& \dot{p}_{r}=U(t, \theta, z, t)+\alpha \sin \psi, \\
& p_{\theta}=r^{2} \Omega(r, \theta, z, t)+\beta \cos \psi, \\
& \alpha \approx \beta=\frac{\delta r}{r} \sim \varepsilon .
\end{aligned}
$$

Differentiating these, substituting into equations (II.3)-(1I.4) give,

$$
\begin{aligned}
& \dot{p}_{r}=\frac{\partial U}{\partial t}+(U+\alpha \sin \psi) \frac{\partial U}{\partial r}+\left(\Omega+\frac{\beta}{r^{2}} \cos \psi\right) \frac{\partial U}{\partial \theta}+ \\
& +\dot{z} \frac{\partial U}{\partial z}+\dot{\alpha} \sin \phi+\alpha \dot{\psi} \cos \psi \\
& =-\frac{\partial V}{\partial r}+\Omega^{2} r+\frac{2 \beta \Omega}{r} \cos \psi+\frac{\beta}{r^{3}} \cos ^{2} \psi \text { s } \\
& -\frac{\partial V}{\partial \theta}=\dot{p}_{e}:=\frac{\partial r^{2} \Omega}{\partial t}+(\tau+d \sin \dot{\psi}) \frac{r \nu^{2}}{\partial \Omega}+\left(\Omega+\frac{\beta}{r^{2}} \omega \operatorname{si}\right) \frac{\partial r^{2} \Omega}{\partial \theta}(1) \\
& +\dot{z} \frac{\partial r^{2} \Omega}{\partial z}+\dot{\beta} \cos \phi-\beta \dot{\psi} \sin \phi,
\end{aligned}
$$


where $(r, \theta, z, t)$ is the generalized betatron frequency

$$
\nu^{2}(r, \theta, z, t) \equiv \frac{1}{r^{3}} \frac{\partial}{\partial r}\left[r^{4} \Omega^{2}(r, \theta, x, t)\right] .
$$

Solving these for: gives

$$
\begin{aligned}
& \dot{\psi}=-\frac{\cos \psi}{\alpha}\left[\frac{\partial V}{\partial r}-\Omega^{2} r+\frac{D U}{I t}\right]+\frac{\sin \psi}{\beta}\left[\frac{\partial V}{\partial \theta}+\frac{D r^{2} \Omega}{D t}\right] \\
& +\cos ^{2} \psi\left[\frac{2 \beta \Omega}{\alpha r}-\frac{\beta}{\alpha r} \frac{\partial U}{\partial \theta}\right]+\frac{\alpha}{\beta} \frac{r \nu^{2}}{2 \Omega} \sin ^{2} \psi \\
& +\frac{1}{2}\left[\frac{d}{d t} \ln \frac{\beta}{\alpha}+\frac{\partial \Omega}{\partial \theta}-\frac{\partial V}{\partial \theta}\right] \sin 2 \psi+\frac{\beta^{2}}{\alpha r^{3}} \cos ^{3} \psi \text {, }
\end{aligned}
$$

where $D / D t=(a / a t+4 a / a r+b z / 3 \theta+2 a / 32)$. In this equation, $U$ and $a$ are not yet specified, although we can view them as representing a radial drift and an averaged dzimuthal rotation frequency. We now chose

$$
\begin{gathered}
\Omega^{2}(r, 0, z, t)=\frac{1}{r} \frac{\partial V}{\partial r}(r, \theta, x, t), \\
V(r, \theta, z, \dot{z}, t)=-\frac{\partial \Omega}{r \nu^{2}}\left[\frac{\partial V}{\partial \theta}+\frac{\partial r^{2} \Omega}{\partial t}+\Omega \frac{\partial r^{2} \Omega}{\partial \theta}+\dot{z} \frac{\partial r^{2} \Omega}{\partial z}\right],
\end{gathered}
$$

so that equation (11.13) begins with a term of 0 (1) rather than order

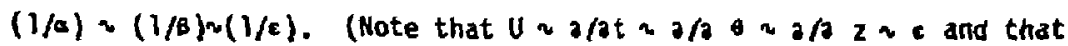
aU/at $\left.+2 U / 2 \theta+2 U / a 2 \sim \varepsilon^{2}\right)$. This $O(1)$ coefficient of $i$ becomes independent of if we choose

$$
\frac{\beta}{\alpha}=\frac{r \nu}{2 \Omega}
$$


As we will imnediately find, these choices are crucial in making a simple drift-kinetic equation with the properties stated above in Section I. Now

$$
\begin{aligned}
\dot{\psi}= & \nu-\frac{\nu}{4 \Omega r} \frac{\partial U}{\partial \theta}-\frac{\operatorname{Ls} \psi}{\alpha}\left[\frac{\partial U}{D t}-\frac{3 \alpha^{2} \nu^{2}}{16 \Omega^{2} r}\right]-\frac{\nu \cos 2 \psi}{4 \Omega r} \frac{\partial U}{\partial \theta} \\
& +\frac{1}{2}\left[\frac{d}{d t} \ln \frac{\rho}{\alpha}+\frac{\partial \Omega}{\partial \theta}-\frac{\partial U}{\partial r}\right] \sin 2 \psi+\frac{\alpha \psi^{2}}{16 \Omega^{2} r} \cos 3 \psi
\end{aligned}
$$

exhibiting the $\&$ behavior we expect for the phase of the betatronvortex oscillations. From equations (II.10), (II.11), (1I.14)-(II.16), we $f$ ind

$$
\begin{aligned}
\frac{d Q_{t} \alpha}{d t}= & \frac{1}{2} \frac{d}{d t} \ln y-\frac{1}{2}\left[\frac{\partial U}{\partial t}-\frac{\alpha^{2} y^{2}}{16 \Omega^{2} r}\right] \sin \psi \\
& -\frac{\nu \sin 2 \psi}{\partial r \Omega} \frac{\partial U}{\partial \theta}+\frac{1}{4}\left[\frac{\partial U}{\partial r}-\frac{\partial \Omega}{\partial \theta}\right] \cos 2 \psi+\frac{d \nu^{2}}{32 r \Omega^{2}} \sin \psi .
\end{aligned}
$$

vlasov's equation for the distribution function $F(r, \theta, a, \psi, z, \dot{z}, t)$

can be written as

$$
\begin{aligned}
\frac{\partial F}{\partial t} & +(U+\alpha \sin \psi) \frac{\partial F}{\partial r}+\left(\Omega+\frac{\nu \alpha}{\partial \Omega r} \cos \psi\right) \frac{\partial F}{\partial \theta}+\dot{\alpha} \frac{\partial F}{\partial \alpha} \\
& +\dot{\psi} \frac{\partial F}{\partial \psi}+\dot{z} \frac{\partial F}{\partial z}-\frac{\partial V}{\partial z} \frac{\partial F}{\partial p_{z}}=0
\end{aligned}
$$

where the foms of $:$ and were already recorded. Following Chew, et a\}. \{9\}, we expand

$$
F=\sum_{j=0}^{\infty} \epsilon^{j-2} F_{j},
$$

which begins with a term of $0\left(\varepsilon^{-2}\right)$ because the number density

$$
n=\int F_{0} \frac{1}{r} d p_{r} d p_{0} d \dot{z}=\int F_{0} \alpha d \alpha d \psi d \dot{z}
$$

is $0(1)$. (Hote that $\alpha<2 \pi<\varphi$ and $0<0<-,-<\dot{2<-)}$ ) and that $F_{j}$ have zero average over if $j \neq 0$ ). The lowest order contribution of equation (II.19) is 


$$
\frac{\partial F_{e}}{\partial \psi}=0
$$

or $F_{0}(r, A, a, z, i, t)$ only and is independent of $\psi$. The next order equation, after averaging over $*$, gives the orift kinetic equation

$$
\frac{\partial F_{0}}{\partial t}+U \frac{\partial F_{0}}{\partial r}+\Omega \frac{\partial F_{0}}{\partial \theta}+\dot{z} \frac{\partial F_{c}}{\partial z}-\frac{\partial V}{\partial z} \cdot \frac{\partial F_{0}}{\partial z}=0=
$$

where we have taken advantage of the fact that to this order, we have an adiabatic invariant,

$$
\frac{d \mu}{d t}=0, \mu \equiv \frac{\alpha^{2}}{\nu}
$$

and defined $F_{0}(r, \theta, \mu, z, \dot{z}, t)$ in equation (II.23).

For axisymetric problems, this drift kinetic equation now involves four parameters $(r, z, \dot{z}, t)$ while $U(r, z, \dot{z}, t)$ is a known quantity according to equations (II.12), (11.14)-(11.15). Equation (11.23) is substantially simpler than the original vlasov equation because the rapid betatronvortex oscillations are averaged out. This is advantageous for numerical simulations because the time-step now follows the macroscopic time relevant to the bunching process rather than the microscopic one involving detailed betatron oscillations. If the full particle orbits are followed in particle code simulations, there is always the danger that the relevant smooth siow drifts on the bunching time may be coarseiy calculated hecause it represents only the residual of tine rapid betatron and circular oscillations. 
I1. The Adjabatic Regine

The drift kinetic equation (II.23) is useful on its own merits when one desires more accurate kisetic information in the longitudinal direction but is satisfied with a first approximation in the transverse direction in order to determine the dependence of self-consistent force fielos from bulk motion. The nature of the approximation of transverst motions can be seen by caiculating averaged "hydrodynamic velocities" $\left(v_{r}, v_{\theta}, v_{2}\right)$ from the $F_{0}$ as determined by equation $(1 I .23)$. Thus,

$$
\begin{aligned}
& v_{r} \simeq\left\langle U+(\mu \nu)^{\frac{1}{2}} \sin \psi\right\rangle_{F_{b}}=U\left(r, \theta, z, v_{z}, t\right)_{2} \\
& v_{0} \simeq\left\langle\Omega r+\frac{\mu^{\frac{1}{2}} \nu^{\frac{3}{2}}}{2 \Omega} \cos \psi\right\rangle_{F_{0}}=\Omega r, \\
& v_{2} \equiv\langle\dot{z}\rangle_{F}=\langle\dot{z}\rangle_{F_{E}},
\end{aligned}
$$

where

$$
\langle h\rangle_{F} \equiv \frac{y^{2}}{4 \Omega n} \int h F d \mu d \psi d \dot{z},
$$

and the numither density $n$ of particles is,

$$
n \equiv \int F \frac{1}{r} d p_{r} d p_{\theta} d \dot{z}=\frac{\nu^{2}}{4 \Omega n} \int F_{\theta} d \mu d \psi d \dot{z}
$$


From this calculation, we see that while no particular approxination has been made to $\langle z\rangle=v_{z}$ the transverse hydrodymamic velocities are those corresponding to $v_{r} \approx U, v_{\theta} \approx$ ar which in fact is correct to the lowest order in $\varepsilon$. However, if we examine these velocities, they correspond to determining $v_{\theta}$ by force balance between centrifugal force and $(-a y / 6 r)$ while $v_{r}$ is determined from $v_{\theta}$ by $p_{\theta}=d\left(\Omega r^{2}\right) / d t$. Thus, effects of fluid stresses and radial inertia term are omitted (because they are ligher order in $c$ ).

In the analogous plasma problem with axial magnetic fields, Chew, Goldberger and Low [9] have cleverly combined equation (1I.23) with the exact continuity and momemtum moments of Vlasov's equation to obtain an even better approximation. Namely, in their viewpoint, equation (11.23) is used only to provide information to evolve the stress tensor $\underline{\underline{p}}$ in time, thus providing closure for the two exact continuity and momentum equations. Within this viewpoint, we calculate the components $\mathrm{P}_{\mathrm{rr}}$ $P_{r \theta}, P_{6 \theta}$, etc., of the stress tensior $\underline{P}$.

$$
\begin{aligned}
& P_{r r}=n\left\langle\mu \nu \sin ^{2} \psi\right\rangle_{F_{0}}=\frac{1}{2} n\langle\mu \nu\rangle_{F_{0}} \\
& P_{\theta \theta}=n\left\langle\mu \frac{\nu^{3}}{4 \Omega^{2}} \cos ^{2} \psi\right\rangle_{F_{0}}=\frac{\nu^{2}}{4 \Omega^{2}} P_{r r} \\
& P_{r \theta}=P_{\theta r}=n\left\langle\frac{\mu \nu^{2}}{4 \Omega} \sin 2 \psi\right\rangle_{F_{0}}=0 \\
& P_{r z}=P_{2 r}=n\left\langle(\mu \nu)^{\frac{1}{2}} \sin \psi\left(\dot{z}-v_{z}\right)\right\rangle_{F_{0}}=0
\end{aligned}
$$




$$
\begin{gathered}
P_{\theta 2}=P_{2 \varepsilon}=n\left\langle\frac{\mu^{\frac{1}{2}} \nu^{\frac{3}{2}}}{2 \Omega} \cos \psi\left(\dot{z}-\frac{s}{2}\right)\right\rangle_{F_{0}}=0 \\
P_{2 z}=n\left\langle\left(\dot{z}-\frac{v}{z}\right)^{2}\right\rangle_{F_{0}}
\end{gathered}
$$

which could all be determined once $F_{0}$ is known.

The complete system of equations of the adiabatic approximation now comprises of the exact cont inuity and mornentum moment equations

$$
\begin{aligned}
& \frac{\partial n}{\partial t}+\frac{1}{r} \frac{\partial}{\partial r}\left(r n v_{r}\right)+\frac{1}{r} \frac{\partial}{\partial \theta}\left(n v_{\theta}\right)+\frac{\partial}{\partial z}\left(n v_{2}\right)=0, \\
& \frac{d v_{r}}{d t}=\frac{\partial v_{r}}{\partial t}+\psi_{r} \frac{\partial v_{r}}{\partial r}+\frac{v_{\theta}}{r} \frac{\partial v_{r}}{\partial \theta}+v_{z} \frac{\partial v_{r}}{\partial z} \\
& =\frac{r_{E}^{2}}{r}-\frac{\partial V}{\partial r}-\frac{1}{n m}\left[\frac{\partial P_{r r}}{\partial r}+\frac{1}{r}\left(P_{r r}-P_{\theta 0}\right)\right] \text {, }
\end{aligned}
$$

$$
\frac{1}{r} \frac{d r v_{0}}{d t} \equiv \frac{\partial v_{\theta}}{\partial t}+v_{r} \frac{\partial v_{\theta}}{\partial r}+\frac{v_{\theta}}{r} \frac{\partial v_{\theta}}{\partial \theta}+\frac{\partial v_{2}}{\partial z}+\frac{1}{r} v_{r} v_{\theta}=-\frac{\partial V}{\partial \theta}, \text { (III.14) }
$$

$$
\frac{d v_{z}}{d t} \equiv \frac{\partial v_{z}}{\partial t}+v_{r} \frac{\partial v_{z}}{\partial r}+\frac{v_{p}}{r} \frac{\partial v_{z}}{\partial \theta}+\frac{v_{z}}{\partial z} \frac{\partial v_{z}}{\partial z}=-\frac{\partial V}{\partial z}-\frac{1}{n m} \frac{\partial P_{22}}{\partial z} \text {, (111.15) }
$$


together with the adiabatic drift equation (II.23) which cetermines the relevant stresses through (III.6), (III.7), (III.11) with subsidiary relations (1I.12), (11.14), (I1.15). (Instead of equation (11.12) for $\checkmark$ we could instead use (IV.B) derived below).

In these equations of the adiabatic regime, the rapid radial and corresponding azimuthal oscillations of jndividual particle orbits have been averaged to give the stress contributions $P_{r r}$ and $P_{\theta \theta}$, but some motions of the betatron timescales are retained in the smooth circulation of fluid motion. As we discussed in Paper I, typical accelerator beam bunckes with anhamonic space charge forces could be represented by two superposed but counter-rotating streams of roughly equal densities. Finally, we note that in addition to our nbvious absence of axial magnetic fields, our results differ from chew, et al.[9] also in that $P_{\theta \theta} / P_{r r}=v^{2} / 4 a^{2}$ may be nearly unity but deviates more and more from unity when anharmonic space charge effects become important.

\section{The Adiabat ic Hydrodynamic Approximation}

In the induction linac for heavy-ion fusion, the particies in a beam bunch traverses the length of the bunch in a time comparable to or slightly less than the total time spent in the accelerator; but there are many betation-vortex oscillations in this same period. The diversity of time scales is one consequence of the fact that the ratio of beam radius over length is $\mathrm{R}_{\mathrm{p}} / \mathrm{L} \sim 10 \mathrm{~cm} / 5 \mathrm{~m} \sim 1 / 50$. On the other hand, we intend to use the "warm-beam" approximation [8] even when $c S_{1 / 3}$. Thus, we can in fact justifiably say that $\left(R_{p} / L\right) \leq 0\left(\varepsilon^{2}\right)$. If the longitudinal disturbances also have length scales $\lambda$ such that $\left(R_{p} / \lambda\right)=0\left(\varepsilon^{2}\right)$ then relative to our implicit order of $(a / a r)=0(1)$ we can order all $(a / a z)=0\left(c^{2}\right)$ 
rather than $O(\varepsilon)$ as $U E$ in in previous sections. Under this latter ordering, we effectively diminish the "heat flow" terms such as $\left\langle\left(i-v_{z}\right) \mu\right\rangle$ and $\left\langle\left(f-v_{z}\right)^{3}\right\rangle$ by as much as one order in $\varepsilon$. They can then be neglected, and we have an adiabatic (fully) hydrodynamic approximation whol the stress components $\mathrm{P}_{\mathrm{rr}}, \mathrm{P}_{22}$ are determined in terms of the lower moments. For exemple, we find [cf. also Ref. 12].

$$
\frac{d}{d t}\left(\frac{P_{r r}}{n}\right) \equiv\left(\frac{\partial}{\partial t}+v_{r} \frac{\partial}{\partial r}+\frac{v_{\theta}}{r} \frac{\partial}{\partial \theta}+v_{2} \frac{\partial}{\partial z}\right)\left(\frac{P_{r r}}{n \nu}\right)=0 \text {, }
$$

$\frac{d}{d t}\left(\frac{P_{z} z}{n}\right) \equiv\left(\frac{\partial}{\partial t}+v_{r} \frac{\partial}{\partial r}+\frac{v_{\theta}}{r} \frac{\partial}{\partial \theta}+v_{z} \frac{\partial}{\partial z}\right)\left(\frac{P_{2 z}}{n}\right)=0$

$$
P_{\theta \theta}=\frac{\nu^{2}}{4 \Omega^{2}} P_{r r}
$$

Actually, the $\partial / \partial z$ terms are smaller in this ordering but are kept for completeness of correspondence to equations (III.12)-(III.15) which are exact. Thus, we do not make any approxinations in the continuity and momentuff equations, but the only approxinations are in those closure equations (IV.1)-(IV.3) which determine she time evolution of the stresses. 
Equation (IV.3) is the same as (III.I) and follows from the discussions of Section III. To derive equation (Ii.1), we nultiply the orift-kinetic equation (II.23) by $\mu$ and integrate over $\mu, \downarrow$ and $\vec{z}$ (i.e. over velocity space). Term by tern we get

$$
\frac{\partial}{\partial t} \int F_{0} \mu d \mu d \psi d \dot{z}=\frac{\partial}{\partial t}\left(\frac{P \Omega}{\nu^{3}} P_{r r}\right)
$$

while,

$$
\begin{aligned}
& \int U(r, \theta, z, i, t) \frac{\partial F_{0}}{\partial r} \mu d \mu d \psi d \dot{z} \\
& =U\left(r, \theta, z, v_{z}, t\right) \frac{\partial}{\partial r} \int F_{0} \mu d \mu d \psi d \dot{z}-\frac{\partial \Omega r}{\nu^{2}} \frac{\partial \Omega}{\partial \sigma} \int\left(\dot{z}-v_{2}\right) \frac{\partial F_{0}}{\partial r} \mu d \mu d \psi d \dot{z} \\
& \simeq v_{r} \frac{\partial}{\partial r}\left[\frac{8 \Omega}{\nu^{3}} P_{r r}\right]
\end{aligned}
$$

according to relation (11I.1), (111.4), (111.6) and the fact that the $20 / 0 z$ term is one order smaller in 6 (note that a heat flux contribution is involved in this higher order term). Also, we have

$$
\int \Omega \frac{\partial F_{\theta}}{\partial \theta} \mu d \mu d \psi d \dot{z}=\Omega \frac{\partial}{\partial \theta}\left(\frac{P \Omega}{p^{3}} P_{\pi}\right) \geq \frac{v_{\theta}}{\gamma} \frac{\partial}{\partial \theta}\left(\frac{8 \Omega}{\nu^{3}} P_{r r}\right),
$$

because of equations (III.2), (III.4) and (III.6). Together, equations (II.23) with $(a / a z)=0\left(\varepsilon^{2}\right)$ and equations (IV.4)-(IV.6) imply that 


$$
\frac{d}{d t}\left(\frac{\Omega P_{r r}}{v^{3}}\right) \equiv\left(\frac{\partial}{\partial t}+v_{r} \frac{\partial}{\partial r}+v_{t} \frac{\partial}{\partial \theta}+v_{z} \frac{\partial}{\partial z}\right)\left(\frac{\Omega P_{r r}}{\nu^{3}}\right)=0 .
$$

The continuity equation (1Il.12) with equations (11.15), (III.1)-(III.3) implies that

$$
\frac{d}{d t}\left(\frac{v^{2}}{4 \Omega}\right)=0
$$

so that equations (IV.i) follows. It is interesting to rote that if in the above derivation we hau only omitted the heat flux tems but not all a/az terms, we would have had instead of equations (IV.7)-(IV.8)

$$
\frac{d \ln }{d t}\left(\frac{\Omega P_{r r}}{\nu^{3}}\right)=\left[\frac{\partial \Omega r}{\gamma^{2}} \frac{\partial \Omega}{\partial z} \frac{\partial v_{z}}{\partial r}-\frac{\partial v_{z}}{\partial z}\right]=\frac{d \ln }{d t}\left(\frac{n \Omega}{v^{2}}\right),
$$

which again implies (IV.l). This raises the possibility that equation (IV.I) might be alternately justifiable by assuming that the fluctuating force $F_{f_{l}}$ mentioned in Section $I$ is suffic int to inhibit longitudinal heat flux, without being so strong as to imply isotropic stresses.

The adiabatic relation (IV.2) follows in similar fashion by multiplying equation (11.23) with $\dot{z}^{2}$ and integrate over $\mu, \psi$ and $\dot{z}$, noting also equation (IV.8) and the fact that relation (III.15) is $d v_{z} d t \simeq 0\left(\varepsilon^{2}\right) \simeq 0$. 
References:

[1] ERDA Summer Study of Heayy Ions for Inertial Fusion, Eds. R. 0. Bangerter, H. B. Herrmansfeldt, and I. Smith, Lawrence Berkeley Laboratory, Report No. LBL-5534 (1976).

[2] Proceedings of the 3977 Heaky lon Fusion Workshop, Ed. L. W. Smith, Brookhaven National Laboratory, Repori No. BNL 50769 (1977).

[3] Proceedings of the 1978 Heavy lon Fusion Workshop, Ed. R. C. Arnoly, Argonne National Laboratory, Repori No. ANL-79-41 (1978)-

[4] Proceedings of the 1979 Heavy lon rusion Workshop, Ed. W. B. Herrmansfeldt, Lawrence Berkeley Laboratory Report No. LBL-10301, Stanford Linear Accelerator Ceater Laboratory, Report No. SL.AC-PUB-2575 (1979).

[5] Mark, J. W-K., "Heayy-Ion inertial Fusion: Target and Bean Propagation" Invited Paper presented at the 22nd annual meet ing of the Div. of Plasma Phys., Am. Physical Soc.; Lawrence Livermore National Laboratory Report No. UCRL-84821 (1980).

[6] Sessler, A.M., "Heavy-Ion Inertial Fusion: The Driver". Invited paper presented at the 22nd aniual meeting of the Div. of Plasma Phys., Am. Physical Soc. (1980). 
[7] Montgonery, D.C. and Tidman, D. A., "Plasma Kinetic Theory", MCGraw Hill sook Co., New rork (1964).

[8] Mark, J. W-K., and Smith, L., "Plasmas in Particle Accelerators: The Warm Bean Equillbria", Lawrence Livermore National Laboratory Report No. UCI018947, Part I (1981).

[9] Chew, G. F., Goldberge:, J. L., and Low, F. E., Los A] amos Lecture Notes on Physics of Ionized Gases, LA-2055 (1955).

[10] Chew, G. F., Goloberger, M. L., and Low, F. '.., Proc. Roy. Soc. (London), Vol. 236, p 112 (1956).

[11] Mark, J. W-K., Krafft, J. and Wang, T. F. "Warm Bean Plasmas: A Hydrodynamic Model of Three Dimensional Instabilities in Particle Accelerators," Maruscript in preparation.

[12] Berman, R. H., and Mark, J. W-K., Astrophys. J., 231, 388 (1979). 